OPEN ACCESS

Edited by:

Tae-Hee Cho,

Hospices Civils de Lyon, France

Reviewed by:

Pierre Seners,

Centre hospitalier Sainte-Anne,

France

Claus Ziegler Simonsen, Aarhus University Hospital, Denmark

*Correspondence:

Xiang Yan Chen

fionachen@cuhk.edu.hk

Specialty section:

This article was submitted to Stroke, a section of the journal

Frontiers in Neurology

Received: 03 February 2017

Accepted: 31 August 2017

Published: 25 September 2017

Citation:

Yang WJ, Fisher M, Zheng L, Niu CB,

Paganini-Hill A, Zhao HL, XU Y,

Wong KS, Ng HK and Chen XY (2017) Histological Characteristics of

Intracranial Atherosclerosis in a

Chinese Population: A Postmortem

Study.

Front. Neurol. 8:488.

doi: 10.3389/fneur.2017.00488

\section{Histological Characteristics of Intracranial Atherosclerosis in a Chinese Population: A Postmortem Study}

\author{
Wen Jie Yang ${ }^{1}$, Mark Fisher 2,3, Lu Zheng', Chun Bo Niu4, Annlia Paganini-Hill'2, \\ Hai Lu Zhao ${ }^{5}$, Yun $\mathrm{Xu}^{6}$, Ka Sing Wong ${ }^{1}$, Ho Keung $\mathrm{Ng}^{7}$ and Xiang Yan Chen ${ }^{1 *}$ \\ 'Department of Medicine and Therapeutics, Chinese University of Hong Kong, Shatin, Hong Kong, ${ }^{2}$ Department of \\ Neurology, University of California Irvine, Irvine, CA, United States, ${ }^{3}$ Department of Pathology \& Laboratory Medicine, \\ University of California Irvine, Irvine, CA, United States, ${ }^{4}$ Department of Pathology, China-Japan Union Hospital Affiliated to \\ Jilin University, Jilin, China, ${ }^{5}$ Center for Diabetic Systems Medicine, Guangxi Key Laboratory of Excellence, Guilin Medical \\ University, Guilin, China, ${ }^{6}$ Department of Neurology, Affiliated Drum Tower Hospital of Nanjing University Medical School, \\ Nanjing, China, ${ }^{7}$ Department of Anatomical and Cellular Pathology, Chinese University of Hong Kong, Shatin, Hong Kong
}

Background: Anterior and posterior circulation atherosclerosis differ in vascular risk factors and stroke mechanisms. However, few studies have compared the pathological features between these lesions. Using a series of intracranial artery specimens, we characterized the intracranial atherosclerotic lesions and compared pathological features among different arteries of the intracranial vasculature.

Methods: Intracranial large arteries of 32 consecutively recruited autopsy cases of Chinese adults aged 45 years or older were examined pathologically using routine histology and immunostaining, to characterize the pathological features of the atherosclerotic lesions. We analyzed middle cerebral arteries (MCAs) (both left and right), vertebral arteries (VAs) (side more affected), and basilar arteries (BAs).

Results: Progressive atherosclerotic lesions were present in 91(71\%) of the 128 arteries examined. Features of complicated plaques were infrequently detected: plaque hemorrhage was encountered in 12\%, neovasculature in 12\%, lumen thrombi in $13 \%$, macrophage infiltration in $20 \%$, and calcification in $25 \%$ of arteries. Luminal narrowing of MCA was the most severe, followed by VA; the BA least stenotic ( $37 \pm 25$ vs. $30 \pm 24$ vs. $20 \pm 20 \%$, all $p<0.05$ ). MCA had more eccentric (vs. concentric) plaques than VA (69 vs. $25 \%, p=0.003$ ) and BA (69 vs. $38 \% ; p=0.03$ ). Lumen thrombi were more frequent in $\mathrm{BA}$, and calcification most commonly occurred in VA atherosclerotic lesions.

Conclusion: Intracranial atherosclerotic plaques were commonly present in this sample, but the lesions generally lacked features of complicated plaques. MCA lesions had demonstrable differences compared with VA and BA lesions. Further studies are needed to determine whether these characteristics indicate a distinctive atherosclerotic phenotype for the intracranial vasculature.

Keywords: intracranial atherosclerosis, pathology, anterior circulation, posterior circulation, intraplaque hemorrhage, thrombus 


\section{INTRODUCTION}

Intracranial atherosclerotic disease (ICAD) is gaining greater clinical prominence as one of the most common causes of ischemic stroke worldwide, especially in Asians $(1,2)$. Current understanding of ICAD has been advanced by several imaging modalities (3), especially high-resolution magnetic resolution imaging (HRMRI) used to visualize intracranial vessel wall pathology (4-6). Although recent advances in HRMRI have made it possible to provide information on abnormalities within the intracranial vessel, use of HRMRI to characterize ICAD with respect to plaque components and vulnerability is limited due to lack of histologic validation (7-11). Better understanding of pathological characteristics of ICAD may have important consequences for interpretation of plaque vessel wall imaging and provide insights into the pathogenesis of ischemic stroke.

Recent studies demonstrated that anatomical location is an important determinant of arterial morphometric characteristics $(12,13)$, and anterior circulation atherosclerosis differs from posterior circulation in prevalence of risk factors and stroke mechanisms (14-17). The purpose of the present study was to investigate histologic features of intracranial atherosclerosis in a series of postmortem specimens taken from Chinese subjects and to identify differences between lesions in the anterior vs. posterior circulation. We hypothesized that substantial differences are identifiable between plaques in the anterior vs. posterior circulation.

\section{PATIENTS AND METHODS}

\section{Participants}

All large intracranial arteries were retrieved from 32 autopsied specimens from December 2003 to June 2005 at the Prince of Wales Hospital, Hong Kong (18), who were indicated for clinical autopsy to investigate the uncertain cause of death. Consent forms were signed by the patients' relatives for performing routine clinical autopsy. Our protocol was approved by the Joint Chinese University of Hong Kong-New Territories East Cluster Clinical Research Ethics Committee (The Joint CUHK-NTEC CREC) for retrospectively collecting the artery specimens from the autopsy cases. The autopsy examination was performed by certified pathologists who were blinded to the aims of this study. Data of clinical characteristics and cause of death were collected from hospital electronic patient records. The causes of death were as follows: CVD (i.e., coronary artery disease, hypertensive heart disease, ischemic stroke, and brain hemorrhage), $n=13$ (41\%); infection or sepsis, $n=3$ (9\%); other natural causes (i.e., hepatitis), $n=13$ (41\%); unnatural causes (i.e., suicides, accidents), $n=3(9 \%)$.

\section{Histological Staining}

Intracranial large arteries [bilateral middle cerebral artery (MCA), bilateral vertebral artery (VA), and basilar artery (BA)] were extracted for each of the 32 subjects. Each artery was divided into three equal parts. Each part was then dissected and decalcified overnight in 10\% formic acid, followed by perfusion fixation in fresh 30\% formaldehyde. Serial sections of the isolated arteries were cut transversely at 4-mm intervals, embedded in paraffin. Bilateral VA, paralleling left and right VA for individual cases, were embedded into one paraffin block together, without special labeling left or right ride. For an individual autopsy case, the side of VA with a more severe degree of atherosclerotic lesions was used in the analysis. Sections $5-\mu \mathrm{m}$ thick were cut and stained with hematoxylin-eosin $(\mathrm{H} \& \mathrm{E})$ and Victoria blue. Immunohistochemical staining was performed to evaluate the degree of macrophage infiltration, using standard avidin-biotin techniques and commercially available antibodies for CD68 (Abcam, Cambridge, MA, USA) at a dilution of 1: 200 .

\section{Plaque Analysis}

Two pathologists (Chun Bo Niu and Hai Lu Zhao), who were blinded to clinical data, examined the histological sections from all segments using standard light microscopy. For each large artery, only the atherosclerotic lesion with the maximum grade of phenotype was subjected to histological and immunohistochemical evaluations. For the 32 autopsy cases, the most stenotic locations from each of the left MCA, right MCA, VA (choosing the artery with more severe lesions), and BA were analyzed for a total of 128 intracranial arteries.

According to the revised American Heart Association criteria (19), the lesions were classified into three groups: (1) disease-free, with normal intima; (2) pre-atherosclerotic intimal lesions showing accumulation of smooth muscle cells (intimal thickening), or luminal accumulation of foam cells (intimal xanthoma); these lesions commonly occur soon after birth and may regress in the later life; (3) progressive atherosclerotic lesions showing extracellular lipid accumulation without necrosis (pathologic intimal thickening), well-formed necrotic core with an overlying fibrous cap (fibrous cap atheroma and thin fibrous cap atheroma), and large areas of calcification (fibrocalcific plaque).

Lesions in which the atherosclerotic plaques were distributed along the entire circumference of the internal elastic laminar were identified as concentric plaques, and those with a disease-free wall present were defined as eccentric plaques, according to prior work (20). The occurrence of plaque complications, including intraplaque hemorrhage (presence of erythrocytes and/or hemosiderin), neovasculature, thrombus, macrophage infiltration, and calcification, were then analyzed and recorded as absent or present.

The histological sections were photographed with a Leica DC 200 digital microscope. The degree of luminal narrowing was measured using Image-Pro Plus according to the method described by Leung et al. (21). The internal elastic membrane in Victoria blue staining was used as the original boundary of the lumen. The internal elastic lamina was traced, and the perimeter of the area bounded by the internal elastic lamina was recorded as " $P$ ". The original luminal area " $A$ " was determined by the formula: $A=P^{2} / 4 \pi$. The area of atherosclerotic plaque was then traced and determined by the software automatically (recorded as "Ai"), and the percentage of luminal narrowing was determined by the formula $(\mathrm{Ai} / A)^{*} 100 \%$. This method eliminated errors due to luminal collapse that might occur during processing. 


\section{Statistical Analysis}

Data analyses were conducted with the SPSS 20.0 software package (SPSS, Inc., USA). Comparisons among MCA, VA, and BA groups were assessed by McNemar's test for categorical data and paired-samples $t$-test for continuous data. A value of $p<0.05$ was considered to be statistically significant.

\section{RESULTS}

\section{General Features of Intracranial Atherosclerosis}

The 32 cases had a median age of 71 years (range $45-97$ years) and most were men $(n=23,72 \%)$. Nine cases $(28 \%)$ were smokers. Hypertension was present in nine cases (28\%) and diabetes in six (19\%). Cardiovascular diseases including ischemic heart disease, ischemic stroke, and hemorrhagic stroke were found in $9(28 \%)$, $14(44 \%)$, and $2(6 \%)$ cases, respectively (Table 1). According to brain MRI and/or autopsy findings, 11 cases showed brain infarct in MCA territories while only 1 case (with atrial fibrillation) had an infarct in posterior circulation territories.

A total of 128 large arteries from 32 autopsy cases were analyzed, including both left and right MCA, the more affected VA, and BA for each patient. Among all the arteries, 37 (29\%) had pre-atherosclerotic intimal lesions, whereas $91(71 \%)$ showed progressive atherosclerotic lesions, including 15 (12\%)

TABLE 1 | Clinical characteristics of 32 autopsy cases.

\begin{tabular}{lc}
\hline & Total $^{*}$ \\
\hline Age (years) & $71[45-97]$ \\
Male & $23(72 \%)$ \\
Smoker & $9(28 \%)$ \\
Hypertension & $9(28 \%)$ \\
Diabetes & $6(19 \%)$ \\
Ischemic heart disease & $9(28 \%)$ \\
Ischemic stroke & $14(44 \%)$ \\
Hemorrhagic stroke & $2(6 \%)$
\end{tabular}

*Median values with the interquartile range, median [IQR] or number of patients with the percentage, $n(\%)$ is shown. pathologic intimal thickening, 72 (56\%) fibrous cap atheroma, and 4 (3\%) fibrocalcific plaque (Figures 1 and 2). Significant atheromatous narrowing of intracranial vessels was infrequently seen, with only 23 segments (18\%) affected by severe atherosclerotic lesions (>50\% luminal narrowing), while 68 arteries (53\%) exhibited moderate stenosis (10-50\% luminal narrowing), with a mean \pm SD stenosis area of $29 \pm 23 \%$. Components of complicated plaques were relatively uncommon, with plaque hemorrhage encountered in $15(12 \%)$, neovasculature in 15 (12\%), lumen thrombus in $17(13 \%)$, macrophage infiltration in $26(20 \%)$, and calcification in 32 (25\%) of 128 arteries (Figure 3). Ulceration and calcified nodule were not detected in any artery.

\section{Differences among MCA, VA, and BA Atherosclerosis}

Plaque morphology and components were compared among MCA, VA and BA. Statistical analysis comparing left and right MCA showed no significant differences. We therefore chose the MCA with more severe lesions for each patient (32 MCAs) and compared the plaque characteristics with 32 VAs and 32 BAs. As shown in (Table 2), the mean area of luminal stenosis rose from $20 \%$ in the BA group to $30 \%$ in VA group and $37 \%$ in MCA group (all $p<0.05)$. Compared with posterior circulation arteries (VA and BA), anterior circulation arteries (MCA) had a higher prevalence of eccentric lesions (69vs. 25\% and 38\%; $p=0.003$ and $p=0.03$, respectively) and a lower prevalcne of lumen thrombi ( 6 vs. $16 \%$, NS and $28 \%, p=0.04$ ). VA plaques showed a higher frequency of calcification compared with BA plaques (41 vs. $16 \%$, $p=0.04$ ). Plaque phenotype did not show significant difference between anterior and posterior circulation arteries. Other components were detected similarly between anterior and posterior circulation plaques.

\section{DISCUSSION}

Based on this postmortem study, the intracranial arteries commonly exhibit atherosclerotic lesions in Chinese adults, but the degree of luminal narrowing is low and features of complicated

TABLE 2 | Comparisons of plaque characteristics among middle cerebral artery (MCA), vertebral artery (VA), and basilar artery (BA) groups.

\begin{tabular}{|c|c|c|c|c|c|c|}
\hline & $\operatorname{MCA}(n=32)$ & VA $(n=32)$ & $\mathrm{BA}(n=32)$ & $p$-Value $\#$ & $p$-Value & p-Value \#\#\# \\
\hline Plaque phenotype & & & & 0.109 & 0.07 & 1.00 \\
\hline $\begin{array}{l}\text { Pre-atherosclerotic intimal } \\
\text { lesions }\end{array}$ & $6(19 \%)$ & $12(38 \%)$ & $12(38 \%)$ & & & \\
\hline $\begin{array}{l}\text { Progressive atherosclerotic } \\
\text { lesions }\end{array}$ & $26(81 \%)$ & $20(62 \%)$ & $20(62 \%)$ & & & \\
\hline Area stenosis & $37 \pm 25 \%$ & $30 \pm 24 \%$ & $20 \pm 20 \%$ & $0.048^{\star}$ & $<0.001^{*}$ & $0.001^{*}$ \\
\hline Distribution & & & & $0.003^{*}$ & $0.03^{*}$ & 0.42 \\
\hline Eccentric & $22(69 \%)$ & $8(25 \%)$ & $12(38 \%)$ & & & \\
\hline Concentric & 10 (31\%) & $24(75 \%)$ & $20(62 \%)$ & & & \\
\hline Intraplaque hemorrhage & $3(9 \%)$ & $5(16 \%)$ & 3 (9\%) & 0.69 & 1.00 & 0.63 \\
\hline Neovasculature & $6(19 \%)$ & $3(9 \%)$ & $3(9 \%)$ & 0.51 & 0.45 & 1.00 \\
\hline Lumen thrombi & $2(6 \%)$ & $5(16 \%)$ & $9(28 \%)$ & 0.45 & $0.04^{*}$ & 0.29 \\
\hline Macrophages & $8(25 \%)$ & $7(22 \%)$ & $7(22 \%)$ & 1.00 & 1.00 & 1.00 \\
\hline Calcification & $9(28 \%)$ & $13(41 \%)$ & $5(16 \%)$ & 0.61 & 0.23 & $0.04^{*}$ \\
\hline
\end{tabular}

"MCA vs. VA; "\#MCA vs. BA; \#\#\#A vs. BA.

${ }^{*} p<0.05$ 


\section{Intimal thickening}

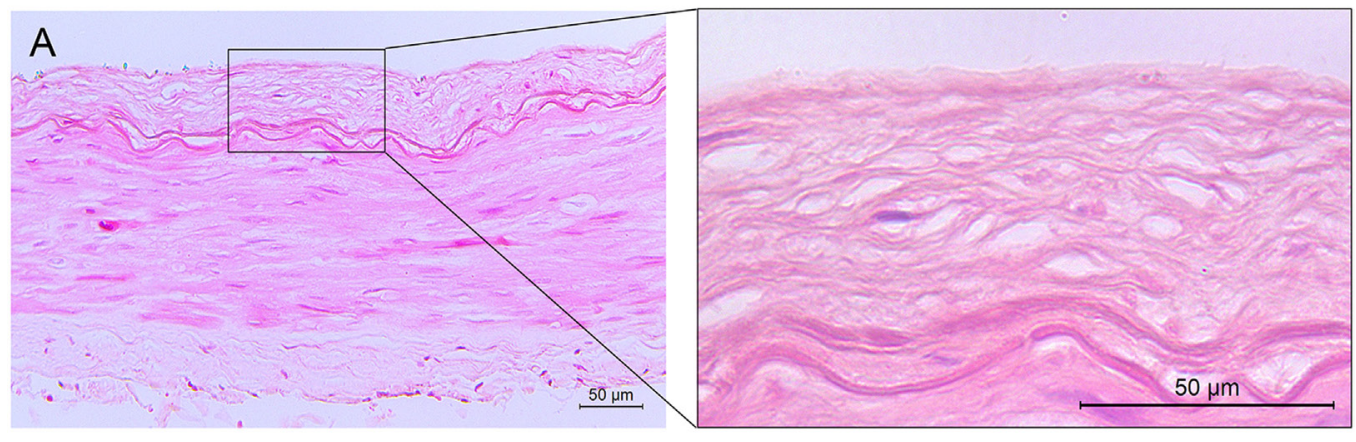

\section{Intimal xanthoma}

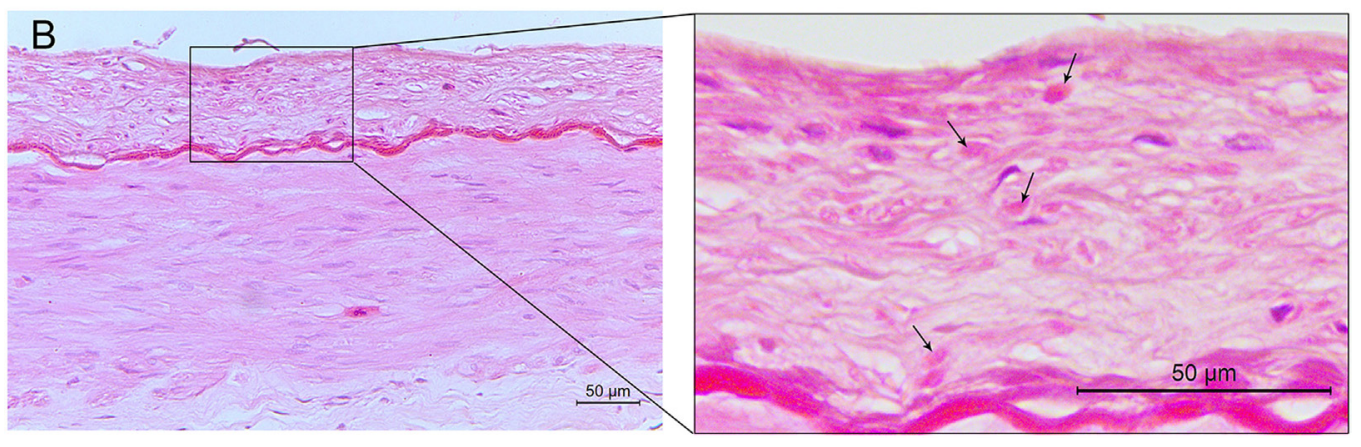

FIGURE 1 | Pre-atherosclerotic intimal lesions. Intimal thickening (A) consists mainly of smooth muscle cells in a proteoglycan-rich matrix. Intimal xanthoma (B) displays intimal thickening with isolated foam cells (arrows). Hematoxylin and eosin (H\&E) staining; original magnification: 10x for original images, 40x for inserted images.

plaques are infrequent. We found that progressive atherosclerotic plaques and eccentric lesions occur more commonly in anterior circulation. The extent of area stenosis varies among large cerebral arteries, with MCA the most severe, followed by VA, and BA the least. Lumen thrombi are more commonly observed in posterior circulation, and calcification is most prevalent in VA plaques.

Previous studies have shown that plaque formation occurs throughout the arteries of Circle of Willies and involves arteries contralateral to brain infarct, although the degree of stenosis, neovasculature, and lipid core were higher in ipsilateral arteries $(18,22-27)$. In the present study, we found that progressive intracranial atherosclerosis was very common in Chinese adults involving $71 \%$ of large cerebral arteries. But the prevalence of intracranial plaques was lower in Caucasian population, with $40 \%$ of ICAS reported in Caucasian patients aged 60 or older (25) and 15\% of advanced plaques in another autopsy studies of 196 Caucasian cases (26).

Although atherosclerotic lesions in intracranial arteries were prevalent, the features of complicated plaque were uncommon (28). A prior autopsy study investigating BA atherosclerosis in patients with a history of stroke depicted a relatively benign histopathological profile, with rare neovasculature, infrequent inflammation, and rare ulceration and rupture (29). Consistent with those findings, we found that plaque complications such as intraplaque hemorrhage, neovasculature, and infiltration of macrophages were less commonly detected when compared with other vascular beds. Also note that plaque ulceration and calcified nodules were not observed in our specimens. Intraplaque hemorrhage was present in only $12 \%$ of intracranial arteries, less than in carotid arteries (19-97\% in symptomatic patients and $7-91 \%$ in asymptomatic patients) $(30,31)$. However, this prevalence in carotid artery studies was primarily in carotid endarterectomy patients, a highly select population with the most severe degree of atherosclerosis. The few studies evaluating intracranial intraplaque hemorrhage using HRMRI have shown substantial variation in its prevalence. Intraplaque hemorrhage was identified in $10 \%$ of high-grade (>70\%) stenotic MCA (32), 17\% of $>50 \%$ stenotic MCA (33), and $42 \%$ of BA (34). Nonetheless, the intracranial intraplaque hemorrhage predicted by MRI has not been validated by standard histology. Note, however, that our autopsy cases were from the general population and few were stroke patients; subjects recruited in HRMRI studies were stroke patients affected by intracranial atherosclerosis. Calcification was prevalent in VA, consistent with the computed tomography findings showing that intracranial VA is the second most common artery affected by calcification after internal carotid artery $(35,36)$.

A notable finding was that luminal stenosis was significantly different among large intracranial arteries, with MCA the most severe, followed by VA, and BA was the least stenotic. This 


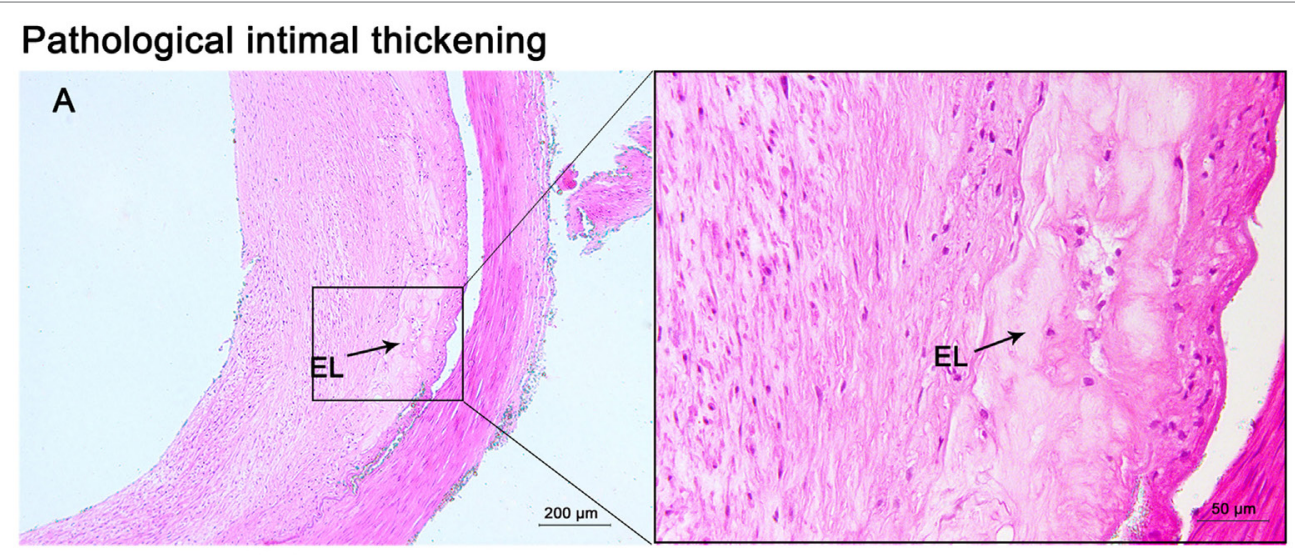

Fibrous cap atheroma

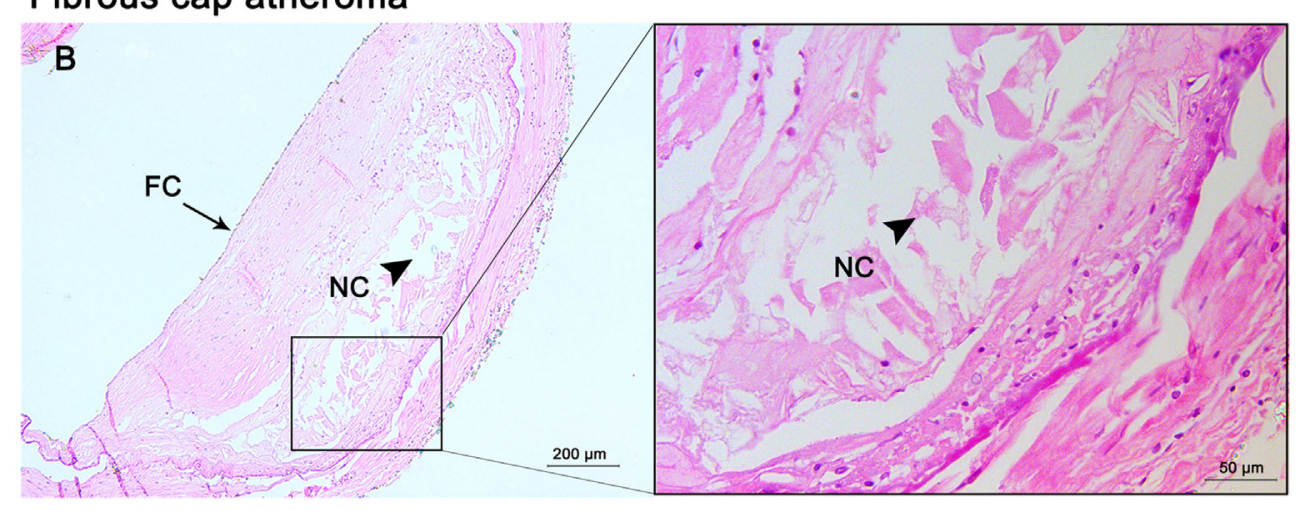

\section{Fibrocalcific atheroma}

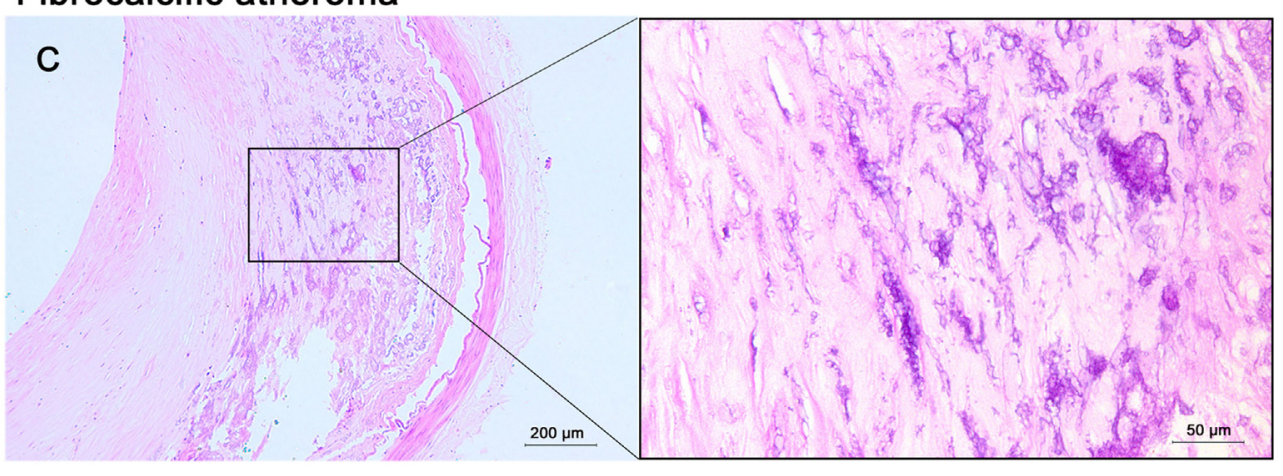

FIGURE 2 | Progressive atherosclerotic lesions. Pathological intima thickening (A) has some extracellular lipid (EL) present deep in the lesion without true necrosis. Fibrous cap atheroma (B) has a well-formed necrotic core (NC) containing lipids with an overlying thick fibrous cap (FC). Fibrocalcific plaques (C) are heavily calcified lesions with or without a necrotic core. Hematoxylin and eosin (H\&E) staining; original magnification: $5 \times$ for original images, $20 \times$ for inserted images.

is consistent with previous reports that MCA was the most commonly affected site by intracranial atherosclerotic lesions in Asians (1, 37, 38). Additionally, we found that the majority of MCA plaques were eccentric lesions, while more VA and BA plaques were concentric. This was consistent with a recent autopsy study in a racially mixed population (39).

This study has some limitations. First, the sample size was relatively small, considering the complexity of atherosclerosis phenotypes and the variations of plaque features between anterior and posterior circulation. Second, we did not include analysis of internal carotid arteries. Finally, plaque features were largely identified based on hematoxylin-eosin staining, which may limit the detection of plaque components.

Intracranial atherosclerosis lesions are commonly present in Chinese adults, with MCA tending to have more severe luminal stenosis and higher prevalence of eccentric lesions. Pathological data of the cerebral arteries may provide reference values for better interpretation of signal changes in HRMRI. Variations of plaque features between anterior and posterior cerebral circulation suggest potentially distinct treatment 

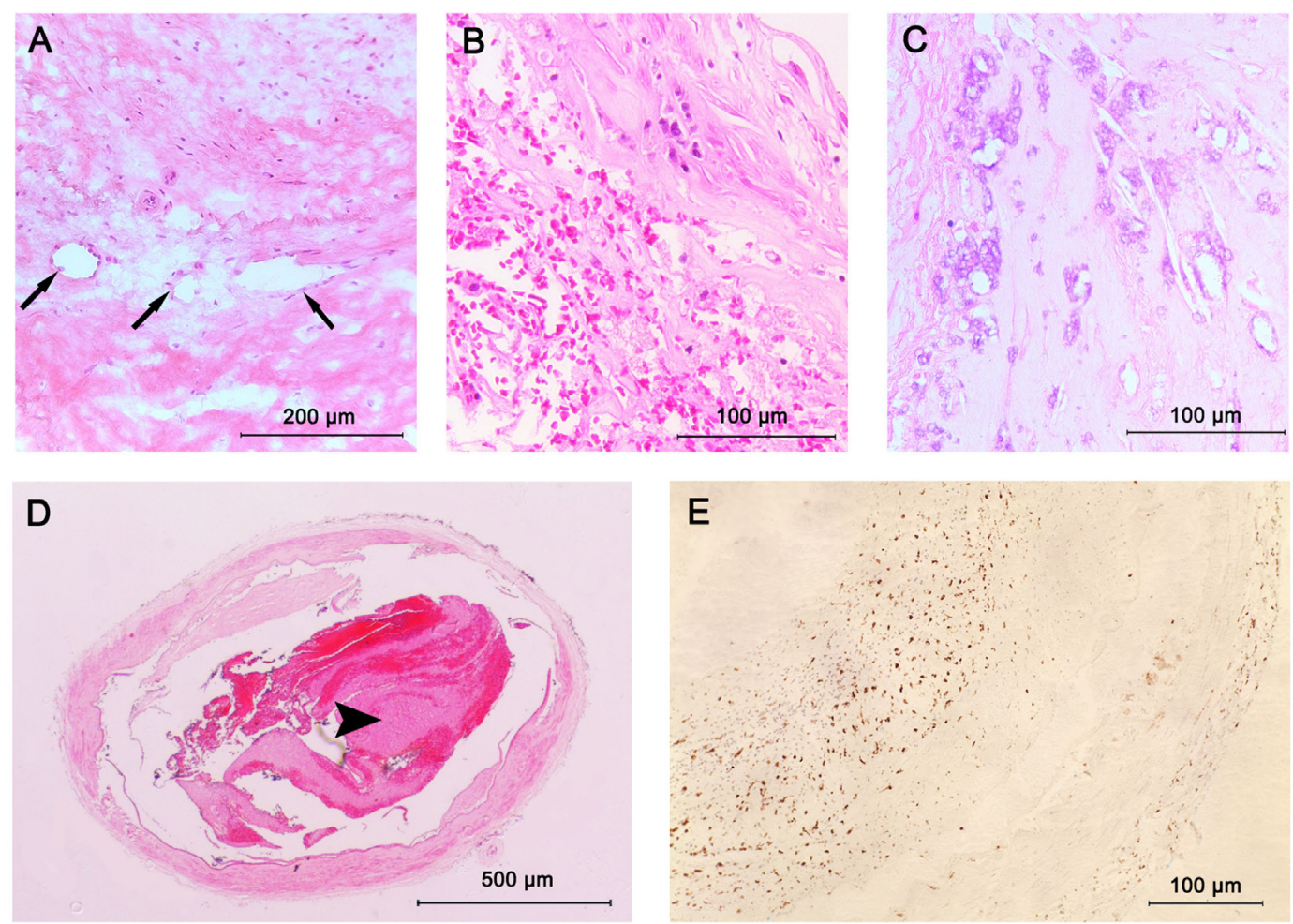

FIGURE 3 | Plaque components in intracranial atherosclerosis. (A) Intraplaque neovasculature (arrows); (B) intraplaque hemorrhage; (C) large areas of calcification seen as purple morula; (D) lumen thrombus (arrowhead); stained with hematoxylin and eosin (H\&E); (E) macrophage infiltration; stained with CD68 antibodies.

strategies for stroke patients with brain infarctions occurring in anterior and posterior circulation. Further studies are needed to confirm whether the observed low frequency of complicated plaques represents a distinct phenotype of intracranial atherosclerosis.

\section{ETHICS STATEMENT}

This study was carried out in accordance with the recommendations of "The Joint Chinese University of Hong Kong-New Territories East Cluster Clinical Research Ethics Committee (The Joint CUHK-NTEC CREC)." The protocol was approved by the "The Joint CUHK-NTEC CREC." Consent forms were signed by the subjects' relatives.

\section{REFERENCES}

1. Caplan LR, Gorelick PB, Hier DB. Race, sex and occlusive cerebrovascular disease: a review. Stroke (1986) 17(4):648-55. doi:10.1161/01.STR.17.4.648

2. Wong KS, Huang YN, Yang HB, Gao S, Li H, Liu JY, et al. A door-to-door survey of intracranial atherosclerosis in Liangbei County, China. Neurology (2007) 68(23):2031-4. doi:10.1212/01.wnl.0000264426.63544.ee

3. Leung TW, Wang L, Soo YO, Ip VH, Chan AY, Au LW, et al. Evolution of intracranial atherosclerotic disease under modern medical therapy. Ann Neurol (2015) 77(3):478-86. doi:10.1002/ana.24340

4. Degnan AJ, Gallagher G, Teng Z, Lu J, Liu Q, Gillard JH. MR angiography and imaging for the evaluation of middle cerebral artery atherosclerotic disease. AJNR Am J Neuroradiol (2012) 33(8):1427-35. doi:10.3174/ajnr.A2697

\section{AUTHOR CONTRIBUTIONS}

WY, LZ, and AP-H were involved in data collection, analysis interpretation, and/or manuscript writing. MF, YX, KW, and XC were responsible for the conception of the study, study design, manuscript writing, and/or final approval. CN and $\mathrm{HZ}$ were responsible for the pathological slides review. HN was responsible for the autopsy examination. All authors approved the final report.

\section{FUNDING}

National Natural Science Foundation of China (Project No. 81371297); National Institutes of Health (NIH) grant (grant number: NS20989); Direct Grant for Research of Chinese University of Hong Kong (Project Code: 4054063).

5. Bodle JD, Feldmann E, Swartz RH, Rumboldt Z, Brown T, Turan TN. High-resolution magnetic resonance imaging: an emerging tool for evaluating intracranial arterial disease. Stroke (2013) 44(1):287-92. doi:10.1161/ STROKEAHA.112.664680

6. Leng X, Wong KS, Liebeskind DS. Evaluating intracranial atherosclerosis rather than intracranial stenosis. Stroke (2014) 45(2):645-51. doi:10.1161/ STROKEAHA.113.002491

7. Kim JM, Jung KH, Sohn CH, Moon J, Han MH, Roh JK. Middle cerebral artery plaque and prediction of the infarction pattern. Arch Neurol (2012) 69(11):1470-5. doi:10.1001/archneurol.2012.1018

8. Chen XY, Wong KS, Lam WW, Ng HK. High signal on T1 sequence of magnetic resonance imaging confirmed to be intraplaque haemorrhage by histology in middle cerebral artery. Int J Stroke (2014) 9(4):E19. doi:10.1111/ijs.12277 
9. Dieleman N, van der Kolk AG, Zwanenburg JJ, Harteveld AA, Biessels GJ, Luijten PR, et al. Imaging intracranial vessel wall pathology with magnetic resonance imaging: current prospects and future directions. Circulation (2014) 130(2):192-201. doi:10.1161/CIRCULATIONAHA.113.006919

10. Yang WJ, Chen XY, Zhao HL, Niu CB, Xu Y, Wong KS, et al. In vitro assessment of histology verified intracranial atherosclerotic disease by $1.5 \mathrm{~T}$ magnetic resonance imaging: concentric or eccentric? Stroke (2016) 47(2):527-30. doi:10.1161/STROKEAHA.115.011086

11. Yang WJ, Chen XY, Zhao HL, Niu CB, Zhang B, Xu Y, et al. Postmortem study of validation of low signal on fat-suppressed T1-weighted magnetic resonance imaging as marker of lipid core in middle cerebral artery atherosclerosis. Stroke (2016) 47:2299-304. doi:10.1161/STROKEAHA.116.013398

12. Gutierrez J, Rosoklija G, Murray J, Chon C, Elkind MS, Goldman J, et al. A quantitative perspective to the study of brain arterial remodeling of donors with and without HIV in the Brain Arterial Remodeling Study (BARS). Front Physiol (2014) 5:56. doi:10.3389/fphys.2014.00056

13. Qiao Y, Anwar Z, Intrapiromkul J, Liu L, Zeiler SR, Leigh R, et al. Patterns and implications of intracranial arterial remodeling in stroke patients. Stroke (2016) 47(2):434-40. doi:10.1161/STROKEAHA.115.009955

14. Subramanian G, Silva J, Silver FL, Fang J, Kapral MK, Oczkowski W, et al. Risk factors for posterior compared to anterior ischemic stroke: an observational study of the registry of the Canadian Stroke Network. Neuroepidemiology (2009) 33(1):12-6. doi:10.1159/000209282

15. Miyamoto $\mathrm{N}$, Tanaka $\mathrm{Y}$, Ueno $\mathrm{Y}$, Tanaka $\mathrm{R}$, Hattori $\mathrm{N}$, Urabe $\mathrm{T}$. Comparison of clinical backgrounds with anterior versus posterior circulation infarcts. J Stroke Cerebrovasc Dis (2010) 19(5):393-7. doi:10.1016/j. jstrokecerebrovasdis.2009.07.012

16. Kim JS, Nah HW, Park SM, Kim SK, Cho KH, Lee J, et al. Risk factors and stroke mechanisms in atherosclerotic stroke: intracranial compared with extracranial and anterior compared with posterior circulation disease. Stroke (2012) 43(12):3313-8. doi:10.1161/STROKEAHA.112.658500

17. Lopez-Cancio E, Matheus MG, Romano JG, Liebeskind DS, Prabhakaran S, Turan TN, et al. Infarct patterns, collaterals and likely causative mechanisms of stroke in symptomatic intracranial atherosclerosis. Cerebrovasc Dis (2014) 37(6):417-22. doi:10.1159/000362922

18. Chen XY, Wong KS, Lam WW, Zhao HL, Ng HK. Middle cerebral artery atherosclerosis: histological comparison between plaques associated with and not associated with infarct in a postmortem study. Cerebrovasc Dis (2008) 25(1-2):74-80. doi:10.1159/000111525

19. Virmani R, Kolodgie FD, Burke AP, Farb A, Schwartz SM. Lessons from sudden coronary death: a comprehensive morphological classification scheme for atherosclerotic lesions. Arterioscler Thromb Vasc Biol (2000) 20(5):1262-75. doi:10.1161/01.ATV.20.5.1262

20. Varnava AM, Mills PG, Davies MJ. Relationship between coronary artery remodeling and plaque vulnerability. Circulation (2002) 105(8):939-43. doi:10.1161/hc0802.104327

21. Leung SY, Ng TH, Yuen ST, Lauder IJ, Ho FC. Pattern of cerebral atherosclerosis in Hong Kong Chinese. Severity in intracranial and extracranial vessels. Stroke (1993) 24(6):779-86. doi:10.1161/01.STR.24.6.779

22. Mazighi M, Labreuche J, Gongora-Rivera F, Duyckaerts C, Hauw JJ, Amarenco P. Autopsy prevalence of intracranial atherosclerosis in patients with fatal stroke. Stroke (2008) 39(4):1142-7. doi:10.1161/ STROKEAHA.107.496513

23. Klein IF, Labreuche J, Lavallee PC, Mazighi M, Duyckaerts C, Hauw JJ, et al. Is moderate atherosclerotic stenosis in the middle cerebral artery a cause of or a coincidental finding in ischemic stroke? Cerebrovasc Dis (2010) 29(2):140-5. doi:10.1159/000262310

24. Majidi S, Sein J, Watanabe M, Hassan AE, Van de Moortele PF, Suri MF, et al. Intracranial-derived atherosclerosis assessment: an in vitro comparison between virtual histology by intravascular ultrasonography, 7T MRI, and histopathologic findings. AJNR Am J Neuroradiol (2013) 34(12):2259-64. doi:10.3174/ajnr.A3631
25. Gutierrez J, Elkind MS, Virmani R, Goldman J, Honig L, Morgello S, et al. A pathological perspective on the natural history of cerebral atherosclerosis. Int J Stroke (2015) 10(7):1074-80. doi:10.1111/ijs.12496

26. Denswil NP, van der Wal AC, Ritz K, de Boer OJ, Aronica E, Troost D, et al. Atherosclerosis in the circle of Willis: spatial differences in composition and in distribution of plaques. Atherosclerosis (2016) 251:78-84. doi:10.1016/j. atherosclerosis.2016.05.047

27. Gutierrez J, Honig L, Elkind MS, Mohr JP, Goldman J, Dwork AJ, et al. Brain arterial aging and its relationship to Alzheimer dementia. Neurology (2016) 86(16):1507-15. doi:10.1212/WNL.0000000000002590

28. Fisher M, Csiba L, Labadzhyan A, Zhou J, Narula N, Narula J. Pathogenesis of intracranial atherosclerosis. Ann Neurol (2012) 72(1):149; author reply 149-150. doi:10.1002/ana.23617

29. Labadzhyan A, Csiba L, Narula N, Zhou J, Narula J, Fisher M. Histopathologic evaluation of basilar artery atherosclerosis. J Neurol Sci (2011) 307(1-2):97-9. doi:10.1016/j.jns.2011.05.004

30. Derksen WJ, Peeters W, van Lammeren GW, Tersteeg C, de Vries JP, de Kleijn DP, et al. Different stages of intraplaque hemorrhage are associated with different plaque phenotypes: a large histopathological study in 794 carotid and 276 femoral endarterectomy specimens. Atherosclerosis (2011) 218(2):369-77. doi:10.1016/j.atherosclerosis.2011.07.104

31. Teng Z, Sadat U, Brown AJ, Gillard JH. Plaque hemorrhage in carotid artery disease: pathogenesis, clinical and biomechanical considerations. J Biomech (2014) 47(4):847-58. doi:10.1016/j.jbiomech.2014.01.013

32. Xu WH, Li ML, Gao S, Ni J, Yao M, Zhou LX, et al. Middle cerebral artery intraplaque hemorrhage: prevalence and clinical relevance. Ann Neurol (2012) 71(2):195-8. doi:10.1002/ana.22626

33. Yang WQ, Huang B, Liu XT, Liu HJ, Li PJ, Zhu WZ. Reproducibility of high-resolution MRI for the middle cerebral artery plaque at 3T. Eur J Radiol (2014) 83(1):e49-55. doi:10.1016/j.ejrad.2013.10.003

34. Yu JH, Kwak HS, Chung GH, Hwang SB, Park MS, Park SH. Association of intraplaque hemorrhage and acute infarction in patients with basilar artery plaque. Stroke (2015) 46(10):2768-72. doi:10.1161/STROKEAHA.115.009412

35. Chen XY, Lam WW, Ng HK, Fan YH, Wong KS. The frequency and determinants of calcification in intracranial arteries in Chinese patients who underwent computed tomography examinations. Cerebrovasc Dis (2006) 21(1-2):91-7. doi:10.1159/000090206

36. Wu XH, Chen XY, Wang LJ, Wong KS. Intracranial artery calcification and its clinical significance. JClin Neurol (2016) 12(3):253-61. doi:10.3988/ jcn.2016.12.3.253

37. Huang YN, Gao S, Li SW, Huang Y, Li JF, Wong KS, et al. Vascular lesions in Chinese patients with transient ischemic attacks. Neurology (1997) 48(2):524-5. doi:10.1212/WNL.48.2.524

38. Dieleman N, Yang W, Abrigo JM, Chu WC, van der Kolk AG, Siero JC, et al. Magnetic resonance imaging of plaque morphology, burden, and distribution in patients with symptomatic middle cerebral artery stenosis. Stroke (2016) 47(7):1797-802. doi:10.1161/STROKEAHA.116.013007

39. Roth W, Morgello S, Goldman J, Mohr JP, Elkind MS, Marshall RS, et al. Histopathological differences between the anterior and posterior brain arteries as a function of aging. Stroke (2017) 48(3):638-44. doi:10.1161/ STROKEAHA.116.015630

Conflict of Interest Statement: The authors declare that the research was conducted in the absence of any commercial or financial relationships that could be construed as a potential conflict of interest.

Copyright $\odot 2017$ Yang, Fisher, Zheng, Niu, Paganini-Hill, Zhao, Xu, Wong, Ng and Chen. This is an open-access article distributed under the terms of the Creative Commons Attribution License (CC BY). The use, distribution or reproduction in other forums is permitted, provided the original author(s) or licensor are credited and that the original publication in this journal is cited, in accordance with accepted academic practice. No use, distribution or reproduction is permitted which does not comply with these terms. 